\title{
FUNDAMENTOS DEL ENFOQUE DE COMPETENCIAS PARA LA VIDA Y DE LA TRANSVERSALIDAD EN EL MINISTERIO DE EDUCACIÓN PÚBLICA
}

\author{
Ingrid Bustos Rojas \\ Viviana Richmond González²
}

\begin{abstract}
Una educación "desde la cuna hasta la tumba, inconforme y reflexiva, que nos inspire un nuevo modo de pensar... Que canalice hacia la vida la inmensa energía creadora que durante siglos hemos despilfarrado en la depredación y la violencia, y nos abra al fin, la segunda oportunidad sobre la tierra"(García, Gabriel, 1994).
\end{abstract}

\section{Resumen}

El presente artículo aborda la visión de mundo, de persona, de conocimiento, de desarrollo humano, de fenómeno educativo, de currículo y de proceso de enseñanza-aprendizaje de las que parte la transversalidad en el Ministerio de Educación Pública (MEP) de Costa Rica. A partir de este fundamento, se presenta la conceptualización de temas transversales, eje transversal y competencias para la vida, como los elementos básicos para el desarrollo de este modelo en el Sistema Educativo Costarricense.

Palabras claves: transversalidad, competencias para la vida, currículo.

\section{Abstract}

This article refers to the vision of the world of the people, knowledge, human development, the educational phenomena, curriculum, and the teaching-learning process from the M.E.P. 's transversality

1 Máster en Educación Primaria con énfasis en Curriculo. Asesora técnica de la División de Desarrollo Curricular del MEP y académica de la División de Educación Rural del CIDE, Universidad Nacional.

2 Licenciada en Educación Especial con énfasis en Retos Múltiples. Integrante de la Comisión Central de Transversalidad del MEP del 2002 al 2005. Se ha desempeñado como docente de estudiantes con necesidades educativas especiales, asesora del Departamento de Educación Integral de la Sexualidad y actualmente funge como Docente de Educación Especial del Equipo Itinerante de la Dirección Regional de Heredia. 
in Costa Rica. Starting with this base; it also presents the conceptualisation of the transversalty, it's topics, cross curricular topics and competences for life; as basic elements for the development of this model in the costarican Educative System.

Keywords: transversality, curriculum, human development.

\section{Introducción}

Como la transversalidad se asume de forma diferenciada en cada uno de los países del mundo, de acuerdo con sus necesidades y características, el presente documento define el enfoque que Costa Rica asume en este sentido.

Dicho enfoque parte de diferentes procesos: primero, una consulta nacional a diferentes instancias iniciada en el año 1998 y, segundo, la construcción teórica de un grupo de profesionales de la División de Desarrollo Curricular del MEP, con apoyo de personas expertas internacionales en la temática en los años 2000 y 2001 y de las apreciaciones, comentarios y sugerencias de docentes en todo el país, que recibieron capacitación sobre transversalidad entre el 2002 y el 2004.

Se establece la transversalidad como un enfoque que permite comprender, analizar y operacionalizar la educación desde una visión humanista, inclusiva e integradora. Este documento contempla el marco filosófico de las distintas concepciones que le dan sustento: la visión de mundo, de persona, del conocimiento del desarrollo humano, del fenómeno educativo, del currículo y del proceso de enseñanza-aprendizaje.

\section{Visión de mundo}

El enfoque de la transversalidad, en concordancia con Delors (1996), reconoce que el mundo está integrado por una pluralidad de ideologías, etnias, culturas, lenguajes, formas de vivir y concebirlo, que crea una serie de tensiones entre lo mundial y lo local, lo universal y lo singular, tradición y modernidad, el largo y el corto plazo, entre la competencia y la igualdad de oportunidades, entre el desarrollo de conocimientos y la capacidad de asimilación del ser humano y entre lo material y lo espiritual.

Esto remite a que en pleno siglo XXI, el mundo no es estático; por el contrario, se encuentra en constante cambio, ya que confluyen en él una gran cantidad de elementos personales y culturales en que la población difiere.

Las costumbres, los valores sociales e individuales, las leyes, las normas de conducta social, el sistema de organización política, las actividades 
productivas, los conocimientos, las creencias religiosas, los logros tecnológicos, las manifestaciones lingüísticas, literarias y estéticas y las formas de recreación, son algunas de las manifestaciones que constituyen, entre otras, el modo de vida cultural que caracteriza cada una de las sociedades del mundo.

Desde el enfoque de transversalidad, la cultura no es vista de manera estática, sino dinámica como la misma sociedad, pues procura afianzar los patrones que tienden a preservar y fortalecer la calidad de vida de las personas y abandona patrones que, en determinado momento, tuvieron vigencia y que luego perdieron su función en relación con los cambios y la evolución del mundo.

\section{Visión de persona}

La transversalidad reconoce a la persona como constructora de su propio destino, con capacidad de elegir dentro de un abanico de posibilidades aquello que considere más pertinente. Es un ser con talentos y capacidades para crear, innovar y responsabilizarse de sí misma.

La persona que se concibe desde este marco tiene la capacidad de mantener sus raíces y al mismo tiempo de participar activamente en la vida de la nación y las comunidades, es un ser integral que posee inteligencias múltiples, capaz de proponerse metas libremente y desarrollar los medios para lograrlas. Es aquel ser provisto de tal potencialidad, que le permite desarrollarse en las tres dimensiones del ser humano: la socioafectiva, la cognoscitiva y la psicomotora en interacción armónica con el ambiente. Tiene, además, una riqueza de vida espiritual (desde su propia comprensión), digna, libre y justa.

Desde el enfoque de la transversalidad, se pretende formar a una persona que interiorice los valores universales, basados en la Declaración de los Derechos Humanos y en los principios permanentes de lo justo, lo racional, lo necesario y conveniente. Una persona que piense y actúe libremente con responsabilidad hacia ella misma, los demás y su medio; con capacidad de alcanzar sus metas y desarrollar un trabajo en torno a objetivos comunes, contribuyendo con sus recursos personales a atender las necesidades del grupo.

\section{Visión del conocimiento}

La transversalidad visualiza el conocimiento y su epistemología desde el paradigma rizomático, el cual se caracteriza por la posibilidad de que los 
saberes se interrelacionen entre sí, mediante la multiplicidad del conocimiento y posibilitando la relación intrínseca entre las distintas áreas del saber.

Podemos, así, tomar la noción de transversalidad y aplicarla al paradigma rizomático del saber: ella sería la matriz de la movilidad por entre los lazos del rizoma, abandonando los verticalismos y horizontalismos que serian insuficientes para un abarcamiento de visión de todo el "horizonte de eventos" posibilitado por un rizoma (Gallo (s.f.), p. 4).

Desde la transversalidad, el conocimiento se visualiza en una construcción constante y con una validez relativa: adaptable y viable en ciertos contextos, pero posiblemente cuestionable en otros. Asimismo, el descubrimiento y aplicación del conocimiento está supeditado a los aspectos de índole valórico y ético, reconociendo en este punto que los grandes avances científicos se han empleado tanto para el fortalecimiento como para la destrucción de la humanidad.

\section{Visión del desarrollo humano}

Desde la transversalidad y según el Foro Mundial sobre la Educación (2000), la educación es el "eje central del desarrollo humano sostenible" (p. 8). Un desarrollo sostenible que centra sus prioridades en el bienestar social de la población mundial, en la búsqueda de la equidad, la justicia y en el acceso igualitario a los servicios existentes.

Como parte de este paradigma, los grandes desafíos que se presentan para la educación de este siglo son: "desarrollo humano sostenible, entendimiento mutuo entre los pueblos y renovación de la democracia efectivamente vivida" (Delors, 1996, p. 16).

El desarrollo humano emerge como un principio organizador aplicable para la mejora de una sociedad, sometida a transformaciones sociales, políticas, tecnológicas, económicas y culturales. En este sentido, debe visibilizarse el abordaje del desarrollo humano desde una perspectiva de equidad de género, que lleva a la construcción de una cultura equitativa que supere los tratos desiguales entre hombres y mujeres, en todos los ámbitos de la actividad humana, para que impere una plataforma de igualdad y equidad.

En educación, los cambios trascendentales acaecidos en la forma de entender el mundo deben ir en una línea paralela a la seguida por la promoción 
de la calidad de vida. Por consiguiente, ha de implementarse un enfoque de desarrollo humano, en el que la satisfacción de la persona, en sus diferentes dimensiones, pase a convertirse en un criterio de máxima relevancia.

\section{Visión del fenómeno educativo}

En las últimas décadas, ha florecido un ideario educativo que se compromete con:

- Una educación a lo largo de toda la vida en el seno de una sociedad equitativa, que aúne la voluntad y la acción de los múltiples agentes y fuerzas educativas (Castellanos y Moyano, 2001, pp. 6 y 7).

Promueve el "aprender a aprender" en una sociedad en constante cambio y que favorece un acercamiento de la institución educativa con la comunidad, con sus necesidades, características y por qué no, con los recursos humanos y materiales con los que cuenta para brindar una educación contextualizada que responda verdaderamente a la demanda social. Con esta visión, el papel de las madres y los padres como primeros educadores y agentes coadyuvantes al proceso de educación formal favorece la intervención integral de las y los estudiante.

- Una educación donde se conjuguen armoniosamente la calidad y equidad de oportunidades para todos y para todas (...) una educación de puertas abiertas a la diversidad, que reconozca, respete y valore la pluralidad de etnias, lenguas, tradiciones y culturas, así como las diferencias individuales, socioeconómicas, de género y generacionales, atendiendo a las necesidades básicas de aprendizaje de las personas (Castellanos y Moyano, 2001, p. 7).

De esta manera, se responde a la doble misión que tiene la educación de "enseñar la diversidad de la especie humana y contribuir a una toma de conciencia de las semejanzas y la interdependencia entre todos los seres humanos" (Delors, 1996, p. 115).

Con esta visión, "no podemos ser forzados a seguir estándares de comportamiento y, en segundo lugar, tales diferencias no obligan a establecer una organización jerárquica de unos grupos humanos sobre otros, sino que más 
bien reconoce que - equitativamente- todas las identidades VALEN"' (Meléndez, 2005, p. 1).

Desde el enfoque de transversalidad, los centros educativos modelan relaciones no sexistas, solidarias, respetando la pluralidad de las condiciones, pensamientos y características personales, que favorecen la crítica constructiva a los sistemas imperantes y sus consecuencias, con decidida voluntad de transformación social.

- Una educación centrada en la formación integral de cada persona, potenciando el desarrollo de competencias para la vida, en función de los cuatro pilares de la educación para el siglo XXI: aprender a conocer, aprender a hacer, aprender a convivir y aprender a ser (Delors, 1996).

En este sentido, el mismo autor establece que "la educación tiene la misión de permitir a todos sin excepción hacer fructificar todos sus talentos y todas sus capacidades de creación, lo que implica que cada uno pueda responsabilizarse de sí mismo y realizar su proyecto personal" (p. 19).

- Una educación que promueva la apropiación de los logros cientificotécnicos y la democratización del acceso a las nuevas tecnologías de la información y la comunicación, en los marcos de estrategias que aseguren su utilización a partir de criterios de calidad y equidad (p. 19).

Si se considera que permanentemente se crean nuevos conocimientos en el mundo, es necesario que la educación y quienes se encuentran en esta rama tengan el compromiso ético-profesional de una actualización permanente, un espíritu investigativo que forme parte intrínseca de su labor.

Asimismo, retoma la necesidad del acceso equitativo a estos conocimientos y a las tecnologías innovadoras, de tal manera que no sean sólo quienes tienen mayores posibilidades económicas, los que se beneficien de ello.

- Una educación de profunda vocación ética sustentada en los valores humanos universales, que contribuya a promover el desarrollo sostenible, la consolidación de los ideales democráticos y la convivencia pacífica y solidaria entre las distintas naciones, pueblos y culturas... (Celorio, 1996, p. 37). 
La transversalidad implica hablar de multiplicidad de modelos de escuela, de la necesidad de contextualizar los centros, de definición plural de intereses y finalidades. Supone asumir una perspectiva globalizadora en la que los problemas se interrelacionen, se interpreten de forma integral, con una orientación capaz de incorporar distintas culturas y visiones en clave de diversidad e igualdad

La educación se visualiza como un proceso que contribuye a asumir el reto ambiental, de forma que propicie un desarrollo que armonice las relaciones entre las personas y la naturaleza, dentro de un marco de respeto por la diversidad cultural, social y étnica. Implica, además, un sentido de responsabilidad de cada ciudadano o ciudadana con respecto a las necesidades de las futuras generaciones.

\section{Visión del currículo}

Desde el enfoque de la transversalidad, el currículo es la integración sistemática de todos los componentes que inciden en el quehacer educativo, para el desarrollo integral de la persona y de la sociedad, en armonía con la naturaleza y consecuente con un desarrollo sostenible.

Así la situación, el currículo es una propuesta intencional, que se ampara en las respectivas legislaciones del país y se programa en forma consensuada para cumplir con los fines y objetivos de la educación; de esta forma, orienta los ideales del ser y del llegar a ser una sociedad autónoma, reflexiva y participativa, conformada por personas competentes en los diversos ámbitos que engloban el desarrollo social, económico, político y cultural del país.

El currículo, desde esta concepción, se caracteriza por ser realista, humanizado, versátil, dinámico, participativo, flexible, práctico, holístico, reflexivo, horizontal, descentralizado, sistemático, racional y consciente; de manera que atienda en forma integral, la formación de los ciudadanos y ciudadanas que el país requiere. Este currículo permite la identificación de los problemas y desafios del contexto en el que se desenvuelve el estudiantado, los factores culturales que deben conservarse y los que necesitan transformarse para la toma de decisiones en materia educativa y curricular.

Un principio de la transversalidad es precisamente la interdisciplinariedad, lo que implica la posibilidad de analizar las diversas situaciones que se presentan en el mundo, desde un abanico amplio de perspectivas. Retoma el elemento ético del conocimiento, de la información y de la formación humana, 
considerando que el eje transversal del sistema educativo costarricense son los "valores".

Un currículo con "un nuevo lenguaje que refiere a la conciliación, al consenso, al holismo, a la resolución pacífica de conflictos, a la solidaridad, a la integralidad, a la inclusión y a la no discriminación entre otros (...) para un desarrollo global, aunque no globalizante, de todos los grupos" (Meléndez, 2005, p. 1).

En definitiva, "el currículo está centrado en el desarrollo de las competencias para la vida más que en el desarrollo memorístico de contenidos" (Rodríguez, 1999).

\section{Visión del proceso de enseñanza-aprendizaje}

El proceso de enseñanza-aprendizaje, desde la transversalidad, retoma los compromisos planteados en el Marco de Acción Regional de Educación para Todos, a saber:

- "Otorgar en las estrategias de mejoramiento de la calidad un lugar central a la escuela y al aula como ambientes de aprendizaje caracterizados por:

- $\quad$ El reconocimiento de la diversidad y heterogeneidad de los estudiantes y la flexibilidad para dar respuestas pertinentes a las necesidades educativas especiales de las y los alumnos.

- El desarrollo de las capacidades para una gestión escolar con autonomía y responsabilidad por sus procesos y resultados" (Foro Mundial sobre la Educación, 2000, p. 9).

Los procesos de enseñanza-aprendizaje parten de la teoría de la construcción del aprendizaje, en concordancia con la Política Educativa hacia el Siglo XXI. Desde esta perspectiva, el estudiantado construye el conocimiento y desarrolla las competencias para la vida, con el apoyo del profesorado, que se convierte en facilitador del proceso.

La práctica pedagógica se fundamenta en los siguientes principios:

a. La afectividad, la motivación, el interés, la disposición, el deseo, la transferencia, clima áulico positivo, son variaciones pedagógicas sobre el mismo eje conceptual que articulan los componentes cognitivos en el proceso de aprendizaje. 
b. La experiencia natural y espontánea como principio de la formación humana, en el sentido de no negar la naturaleza del niño o la niña, del o la adolescente, sino partir de ella, teniendo en cuenta sus necesidades e intereses y permitiendo el despliegue natural de los propios talentos.

c. La formación integral de la persona no se da aislada o por fuera de la cultura y de la sociedad, sino en medio de ella y, en consecuencia, el ambiente sociocultural se puede diseñar como un entorno de aprendizaje que influya en el desarrollo de competencias y su autorrealización.

d. La niña y el niño no son adultos pequeños; su formación hasta la mayoría de edad se desarrolla, en gran parte, por un proceso constructivo interior, progresivo y diferenciado que es necesario que la actividad educativa respete.

e. Con base en la vivencia de diversas experiencias, el niño, la niña, la y el adolescente construyen sus propias herramientas conceptuales y su propio aprendizaje, lo que contribuye con su desarrollo y autoformación.

f. Al lado del o la docente, el estudiantado eleva su potencial de desarrollo en la medida en que se le muestren las posibilidades de acción, horizontes de interrogación y de soluciones hipotéticas que amplían y realizan sus zonas de desarrollo potencial.

g. El elemento lúdico es una herramienta que forma a la persona en relación con los y las demás, con la naturaleza y consigo misma, en la medida en que le permite representar y proyectar las experiencias y aprendizajes significativos de su entorno.

h. El principio de la individualización reconoce las diferencias no sólo orgánicas, sino también de desarrollo cognitivo, de estilo de afrontar y resolver los problemas, diferencias motivacionales, interculturales y experienciales, de necesidades educativas, variedad de proyectos 0 metas personales, oportunidades socioculturales, relaciones e interacciones con el medio natural y social, que van estructurando las diferencias individuales que el profesorado necesita atender para lograr una enseñanza personalizada, como lo postula la pedagogía inclusiva.

Considerando los principios anteriores, el aprendizaje escolar es un acto social, afectivo, emocional, cognitivo, que se da por la acción mediadora de la o el docente y otros agentes educativos. El o la docente, en su función mediadora facilita la construcción de conocimientos, animación del aprendizaje, supervisión y guía del proceso de aprendizaje e investigación educativa. 


\section{Transversalidad}

El enfoque de la transversalidad en el sistema educativo costarricense nace a partir de una visión de mundo pluralista y diversa, de una visión de persona constructora de su propio destino, de una visión del conocimiento desde una perspectiva rizomática, de una visión social del desarrollo humano, de una visión del fenómeno educativo a partir de un ideario educativo, de una visión de currículo integrado, flexible y abierto y de un proceso de enseñanza-aprendizaje centrado en la construcción de competencias para la vida del y la estudiante.

Esta transversalidad implica, por lo tanto, una crítica radical a los componentes de un sistema educativo tradicional y, por ende, a los modelos dominantes basados en la desigualdad y en la injusticia.

En este sentido, la transversalidad critica las propias contradicciones entre el currículo explícito y el implícito, y establece la necesidad de una conexión entre lo escolar y lo extraescolar, replanteando la educación desde una visión ética. Este enfoque fortalece la evaluación como forma de valoración del proceso, para mejorar y generar un cambio positivo, y no para jerarquizar cuantitativamente (MEP, 2004b).

Como parte de este análisis, Costa Rica ha definido la transversalidad como un:

"Enfoque educativo que aprovecha las oportunidades que ofrece el curriculo, incorporando en los procesos de diseño, desarrollo, evaluación y administración curricular; determinados aprendizajes para la vida, integradores y significativos, dirigidos al mejoramiento de la calidad de vida individual y social. Es de carácter holístico, axiológico, interdisciplinario y contextualizado" (MEP, 2004b, p. 2).

De esta manera, se retoma la necesidad de la inclusión de los aprendizajes para la vida en el currículo, explicitada también en el Marco de Acción Regional de Educación para Todos (2002):

"La inclusión de estos aprendizajes como contenidos curriculares, transversales y/o disciplinares, constituye un desafio asociado a la nueva construcción curricular, al trabajo conjunto con la comunidad $y$ al rol del profesor como modelo de las competencias para la vida" (p. 6). 
Los principios metodológicos para una didáctica de la transversalidad y la inclusión de estos aprendizajes son, según Magendzo (2002):

- $\quad$ Principio de integración: fortalecer las vinculaciones con las oportunidades existentes tanto desde el currículo explícito (planes, programas) como desde el currículo implícito (recreos, fiestas, intervenciones espontáneas).

- $\quad$ Principio de recurrencia: reiterar los aprendizajes para la vida en circunstancias distintas y variadas y mediante diferentes estrategias.

- $\quad$ Principio de gradualidad: desarrollar competencias para la vida a partir de la madurez del estudiantado, sus características y desarrollo evolutivo.

- $\quad$ Principio de coherencia: retomar la necesidad de que el discurso sea coherente con la práctica.

- Principio de problematización: plantear y analizar situaciones problemáticas que evidencien tensiones actitudinales y valorativas.

- $\quad$ Principio de apropiación: retomar la observación del propio discurso y de las acciones del estudiantado.

- Principio de participación inclusiva: considerar las diversidades personales y culturales existentes.

Para la evaluación de estos principios, algunos criterios básicos, mencionados por Magendzo (2002), que deben tomarse, se resumen a continuación:

- Una evaluación integrada: apreciar de manera integrada los aprendizajes de contenidos disciplinarios y las dimensiones de desarrollo a que apunta la transversalidad.

- Una práctica colectiva: realizar un esfuerzo conjunto de reflexión y acción de todos los actores del proceso educativo.

- Una evaluación de la acción y de los discursos: atender las acciones en que los y las estudiantes ponen en juego lo aprendido y a las conceptualizaciones discursivas que elaboran.

- Una mirada en perspectiva: reconocer lo "voluble" de estas acciones y discursos, apreciándolos en perspectiva y como expresiones de procesos de aprendizaje en evolución.

- Un enfoque de evaluación interpretativo: observar y apreciar los aprendizajes de las y los estudiantes desde sus vivencias y visiones de la realidad. 
Desde esta perspectiva, se deduce que la transversalidad no se reduce a:

- La vinculación de áreas supuestamente afines, por ejemplo, entre Estudios Sociales y el Tema Transversal de Derechos Humanos o entre la disciplina de Ciencias y el Tema Transversal de Educación para la Salud; por el contrario, las vinculaciones se pueden dar desde cualquier disciplina de estudio a partir de una perspectiva rizomática del conocimiento.

- La incorporación superficial de otras disciplinas de estudio que se añaden a las disciplinas clásicas, invisibilizando la perspectiva interdisciplinaria de la transversalidad.

- Un enfoque exclusivamente valorativo, ya que integra este enfoque y lo complementa con el desarrollo de competencias para la vida, visibilizando los cuatro pilares establecidos por Delors.

\section{Temas transversales}

A partir de las necesidades sociales emergentes, se han establecido los siguientes temas transversales del currículo costarricense, retomados en acuerdo 15-04 del Consejo Superior de Educación: Cultura Ambiental para el Desarrollo Sostenible, Educación Integral de la Sexualidad, Educación para la Salud y Vivencia de los Derechos Humanos para la Democracia y la Paz.

Estos temas no deben visualizarse como compartimentos aislados, de hecho, presentan múltiples interrelaciones entre sí:

Constituyen dimensiones de la educación que impregnan las diferentes materias o asignaturas. No responden a la lógica de una disciplina aislada, sino que atraviesan todo el trabajo curricular, desde el diseño de los objetivos, contenidos, métodos y formas de enseñanza y aprendizaje, hasta el desarrollo del proceso vivo en el aula y la evaluación permanente de los resultados del aprendizaje, la calidad de la intervención pedagógica del profesorado y el propio proyecto curricular elaborado (Castellanos y Moyano, 2001, p. 15).

\section{Eje transversal de valores}

Los valores son el eje transversal del sistema educativo costarricense, además de constituir uno de los elementos de las competencias. En este 
sentido, Delors (1996) retoma la necesidad de "revalorizar los aspectos éticos y culturales de la educación, y para ello dar a cada uno los medios de comprender al otro en su particularidad y percibir el mundo en su curso caótico hacia una cierta unidad" (p. 38).

Es fundamental, por lo tanto, que las y los docentes promuevan todos aquellos valores que supongan el superar las ideologías androcentristas y etnocentristas, y se dirijan al respeto a la diversidad, la equidad, la justicia social, etc. Como establece Meléndez (2005), "un marco axiológico promovido por los principios emanados de la carta universal de los Derechos de las Personas, por una idea real de Democracia y por una ruptura de las posiciones maniqueas" (p. 1).

Para ello, se requiere de una educación efectiva en valores, que "trata de que el alumnado construya sus propios valores y criterios a partir de experiencias interesantes y conocimientos previos que los docentes facilitarán y apoyarán" (MEP, 2004a). De esta manera, no cabe duda que la mejor estrategia para educar en valores es a partir de la cotidianidad del estudiantado, desde sus propias acciones y las de personas en su entorno, discutiendo y analizando la conveniencia o no de las mismas, y las tensiones actitudinales y valóricas que se presentan.

En este punto es importante resaltar el principio de coherencia de la transversalidad, por cuanto los valores se enseñan fundamentalmente con el ejemplo. Una adecuada educación en valores debe reflejarlos "en las acciones programáticas y en las actuaciones docentes y de todo el personal del Ministerio de Educación" (MEP, 2004b).

\section{Competencias para la vida}

Como se ha mencionado, uno de los propósitos fundamentales de la transversalidad es el desarrollo de competencias para la vida, lo que implica el desarrollo de herramientas para enfrentar la vida de forma responsable, autónoma y satisfactoria. Entonces, ser competente significa saber, saber hacer, saber ser y saber convivir, de manera que las capacidades desarrolladas les permitan enfrentar los problemas de la vida cotidiana.

El Ministerio de Educación Pública (2004b) definió competencias como: "un conjunto integrado de conocimientos, procedimientos, actitudes y valores, que permite un desempeño satisfactorio y autónomo ante situaciones concretas de la vida personal y social"' (p. 6). 
Estas competencias analizadas desde el enfoque de transversalidad, se definieron como:

Aquellas que atraviesan e impregnan horizontal y verticalmente, todas las asignaturas del currículo y requieren para su desarrollo del aporte integrado y coordinado de las diferentes disciplinas de estudio, así como de una acción pedagógica conjunta (Castellanos, 2002, en MEP, 2004b, p. 8).

En el momento actual, definimos la competencia como el conjunto de saberes, habilidades, valores y actitudes que permiten a la persona saber, saber aprender, saber hacer, saber ser y saber convivir con los demás para desempeñarse en forma autónoma, acertada y pertinente ante las demandas personales y sociales.

En este sentido, los saberes contemplan todo el cúmulo de conceptos, hechos o procedimientos que la persona requiere saber para desempeñarse de forma eficiente, pertinente, eficaz y precisa.

Las habilidades integran los procesos cognitivos que involucran reflexión y análisis crítico, la inteligencia emocional que permite a la persona identificar y responder constructivamente ante las emociones propias y las de los demás; también refiere a las habilidades comunicativas que facilitan la expresión asertiva, creativa y crítica de sentimientos, pensamientos, emociones y la interpretación de mensajes para la resolución de problemas comunicativos.

Asimismo, los valores estimulan y orientan determinadas prácticas desde la solidaridad, la equidad, el respeto, la honestidad, entre otros, de acuerdo con las condiciones personales, del contexto y de la propia relación con los demás.

Las actitudes se refieren a la forma en que se asumen los retos de la vida. Logran el discernimiento sobre los sentimientos que emanan de un determinado hecho y la intención en la forma particular de proceder y actuar ante ciertas situaciones.

Las competencias, como su misma definición lo indica, tienen una relación estrecha con los planteamientos que realiza Delors acerca de los Pilares de la Educación hacia el Siglo XXI. Esta relación se explica a continuación (Delors, 1996): 
- Aprender a conocer: Uno de los elementos de las competencias son los "saberes", lo que implica dominar los instrumentos mismos del saber y aprender a comprender el mundo que nos rodea. "Aprender para conocer supone, en primer término aprender a aprender, ejercitando la atención, la memoria y el pensamiento" (p. 108).

- Aprender a hacer: Se relaciona con el componente de "habilidades" de la competencia y busca poner en práctica los conocimientos, desarrollar la capacidad de "transformar el progreso de los conocimientos en innovaciones generadoras de nuevos empleos y empresas" (p. 110), "de comunicarse y de trabajar con los demás, de afrontar y solucionar conflictos" (p. 111).

- Aprender a vivir juntos, aprender a vivir con los demás: Están implicados los cuatro elementos, en especial "valores" y "actitudes", ya que promueve una educación de la alteridad que "evite los conflictos o los solucione de manera pacífica, fomentando el conocimiento de los demás, de sus culturas y espiritualidad" (p. 114).

- Aprender a ser: De esta manera, se visualiza la integración de los cuatro elementos de la competencia, contribuyendo al desarrollo global de cada persona: cuerpo y mente, inteligencia, sensibilidad, espiritualidad.

Asimismo, estos pilares son retomados en el Marco de Educación para Todos celebrado en Dakar (2000), al establecer como un objetivo que:

Todos los niños, jóvenes y adultos, en su condición de seres humanos tienen derecho a beneficiarse de una educación que satisfaga sus necesidades básicas de aprendizaje en la acepción más noble y más plena del término, una educación que comprenda aprender a asimilar conocimientos, a hacer, a vivir con los demás y a ser (p. 12).

De igual manera, el Marco de Acción Regional (2000) indica que los países se comprometen a:

- "Continuar los procesos de reforma curricular y fortalecerlos para incluir como contenidos de aprendizaje significativo las habilidades, valores y actitudes para la vida, que alienten a las familias a mantener a sus hijos en la escuela y que doten a las personas de los instrumentos 
necesarios para superar la pobreza y mejorar la calidad de vida de las familias y las comunidades" (p. 7).

- "La educación debe proporcionar habilidades y competencias para vivir y desarrollar una cultura del derecho, el ejercicio de la ciudadanía y la vida democrática, la paz y la no discriminación; la formación de valores cívicos y éticos; la sexualidad; la prevención de la drogadicción y el alcoholismo; la preservación y cuidado del medio ambiente" (p. 12).

\section{Reflexiones finales}

Como muchos de los lectores han intuido a lo largo del artículo, el enfoque de la transversalidad se convierte, desde la lógica expuesta, en un ideal o una meta por alcanzar. En este sentido, para que el sistema educativo esté completamente transversalizado, las instituciones educativas deben desarrollar una propuesta curricular en la que las fundamentaciones, prácticas pedagógicas, estructuras organizativas, sistemas de validación y evaluación y el papel de la comunidad educativa, respondan a las visiones como las que la transversalidad propone (Celorio, 1996).

Para que esto se haga realidad, es imprescindible la determinación y claridad política en relación con estas "simples” preguntas: ¿hacia adónde vamos en educación?, ¿qué perfil de ser humano queremos potenciar en Costa Rica?, ¿realmente educan las instituciones educativas?, ¿realmente los maestros enseñan?, ¿los estudiantes aprenden?

No cabe duda que el camino es largo, pero estamos firmemente convencidas de que aunque sea largo y complejo, es mejor empezar a actuar y a proponer, que quedarse sólo en la identificación de la problemática educativa nacional.

Si bien la transversalidad no es la "panacea" ni integra todos los elementos sujetos de análisis del sistema educativo, se constituye en una ventana, y por qué no, en una discreta síntesis de las construcciones históricas y de los aportes de grandes maestras y maestros. ¡Está en todos nosotros sacarle el provecho que tiene!

Desde esta misma lógica, la transversalidad puede y debe fortalecerse con los aportes socioculturales producto de la evolución en el pensamiento, debe ser, desde el principio de la coherencia, un reflejo del desarrollo de las competencias de las nuevas generaciones. 


\section{Referencias}

Atoréis, A. (2005). Habilidades para la vida en las evaluaciones de lectura y escritura. Buenos Aires.

Castellanos, B. y Moyano, M. (2001). La educación de la sexualidad en países de América Latina y el Caribe. UNFPA.

Celorio, G. (1996). Desde una transversalidad de trámite a una transversalidad renovadora. Aula de Innovación Educativa 1.

Delors, J. (1996). La educación encierra un tesoro. Informe a UNESCO de la Comisión Internacional sobre la Educación para el Siglo XXI. Quito: UNESCO.

Foro Mundial sobre la Educación. (2000). Educación para todos. DAKAR.

Gallo, S. (s.f.). Conocimiento y transversalidad. Brasil: CAMPINAS.

González, O. y Flores, M. (1998). El trabajo docente. México: TRILLAS.

Marco de Acción Regional. (2000). Educación para todos en las Américas. Santo Domingo.

Magendzo, A. (2002). Los temas transversales en el trabajo de aula. San José, Costa Rica.

Meléndez, L. (2005). Diversidad y equidad. Comisión Nacional de Rescate de Valores.

Ministerio de Educación Nacional de Colombia. (2003). Estándares básicos de competencias ciudadanas. Bogotá: IPSA.

Ministerio de Educación Pública. (2004a). Módulo de auto y mutua capacitación para la educación en valores. San José, Costa Rica.

Ministerio de Educación Pública. (2004b). Transversalidad en el Sistema Educativo Costarricense. San José, Costa Rica.

I Jornada de Transversalidad. (1995). Conclusiones de los Seminarios de Transversalidad. Recuperado el 10 de abril de 2002, de http://www.cip.fuhem.es/ EDUCA/docs/ljornada.doc.

Rodríguez, J. L. (1999). Autonomía curricular. Madrid: Consejo Escolar del Estado. 